PHYSICAL REVIEW D 95, 044004 (2017)

\title{
UV completion of the Starobinsky model, tensor-to-scalar ratio, and constraints on nonlocality
}

\author{
James Edholm \\ Consortium for Fundamental Physics, Lancaster University, Lancaster LA1 4YB, United Kingdom
}

(Received 27 November 2016; published 6 February 2017)

\begin{abstract}
In this paper, we build upon the successes of the ultraviolet (UV) completion of the Starobinsky model of inflation. This involves an extension of the Einstein-Hilbert term by an infinite covariant derivative theory of gravity which is quadratic in curvature. It has been shown that such a theory can potentially resolve the cosmological singularity for a flat, homogeneous and isotropic geometry, and now it can also provide a successful cosmological inflation model, which in the infrared regime matches all the predictions of the Starobinsky model of inflation. The aim of this paper is to show that the tensor-to-scalar ratio is modified by the scale of nonlocality, and in general a wider range of tensor-to-scalar ratios can be obtained in this class of model, which can put a lower bound on the scale of nonlocality for the first time as large as the $\mathcal{O}\left(10^{14}\right) \mathrm{GeV}$.
\end{abstract}

DOI: 10.1103/PhysRevD.95.044004

\section{INTRODUCTION}

Einstein's theory of general relativity (GR) has been extremely successful in its predictions about the infrared (IR) regime [1]. However, in the ultraviolet (UV) regime, the theory exhibits pathologies, and at a quantum level the theory becomes nonrenormalizable. Classically, GR allows both black hole and cosmological type singularities. It has been known for some time from a seminal paper by Stelle [2] that quadratic curvature gravity is renormalizable, but in general it suffers from the presence of a Weyl ghost. ${ }^{1}$

In $[4,5]$, it was demonstrated that one can tame the problem of ghosts in a quadratic curvature gravity, provided one invokes infinite covariant derivatives acting on the curvature. ${ }^{2}$ In this case, the graviton propagator is modified by these infinite derivatives, but it is still possible to retain the original massless graviton degrees of freedom without introducing any new poles in either the spin- 0 or spin- 2 component of the graviton propagator. This can be achieved if the propagator is modified by an exponent of an entire function, i.e. $e^{\gamma(\square)}$, where $\gamma(\square)$ is an entire function of the d'Alembertian, $\square=g^{\mu \nu} \nabla_{\mu} \nabla_{\nu}$, where the greek indices run from $0,1,2,3$. The exponential of an entire function contains no roots by construction. If $\gamma>0$, and $\square \rightarrow \infty$, the propagator is even more convergent than a polynomial of finite degree, thus improving upon the

\footnotetext{
${ }^{1}$ This is a generic problem for any higher derivative theory, i.e. more than 2 derivatives, where extra derivatives count for extra poles in the propagator and extra degrees of freedom other than the original degrees of freedom. The extra poles generically harbor ghosts [3].

${ }^{2}$ In [5], the authors constructed the most generic quadratic curvature gravity involving Ricci scalar and tensor, and the Riemann/Weyl, which is ghost free and singularity free around Minkowski background.
}

UV properties [6-9]. In the IR, one recovers the original graviton propagator $[5,10]$.

The quantum UV aspects are also improved, due to the fact that the vertex interactions in such infinite derivative theories, with exponential modification of the propagator in the UV, become nonlocal. The scale of these modifications is governed by the scale of nonlocality $M$. This has been illustrated in thermal aspects of string theory [11], and in an improved higher derivative extension of the electroweak Standard Model [12]. It has also been shown that ultrahigh energy trans-Planckian scatterings in this case do not blow up in the UV [9].

From the classical point of view, in the linear regime the theory has resolved the Newtonian singularity, and the black hole singularity for mini black holes [5], ${ }^{3}$ and also the dynamical formation of such black holes, which have no horizon and no Schwarzschild's singularity [15].

The initial motivation of studying ghost-free infinite derivative gravity (IDG) was to resolve the cosmological big bang singularity problem in Einstein's theory of gravity, by supplanting it with a big bounce [4], and to study cosmological perturbations around the big bounce [16]. In $[17,18]$, it was shown that sub- and super-Hubble perturbations around the bouncing solution are stable. It was already pointed out in $[4,18-20]$ that such a quadratic action of gravity would serve as an UV completion of the original Starobinsky model of inflation [21]. In fact, [19] already mentioned the possibility of explaining low multipoles observed in the temperature anisotropy of the cosmic microwave background radiation (CMBR) [22,23], and its connection with a bouncing cosmology in connection with

\footnotetext{
${ }^{3}$ See also $[13,14]$, where infinite derivative corrections to curvature have been proposed from string theory and string theory. One would expect infinite higher derivative corrections in the gravitational sector, due to $\alpha^{\prime}$ corrections.
} 
a UV-improved Starobinsky inflation. A rigorous proof of avoiding singularity was provided in [24].

Indeed, the scale of nonlocality $M$ is one of the key parameters for any such ghost-free higher derivative modification of gravity. It is important to constrain this parameter from all possible observations. One of the best constraints on $M$ arises from the fact that at table-top experiments it is possible to constrain the departure from the $1 / r$ fall of Newtonian gravity [25], which has been tested up to $5 \times 10^{-6}$ meters, and this places a constraint on the scale of nonlocality of $M>0.004 \mathrm{eV}$ [26]. This is a very weak but useful constraint.

The main aim of this paper is to improve the constraint on the scale of nonlocality, i.e. $M$, from cosmological observations, such as inflationary cosmological perturbations [27].

Primordial inflation [28-30] is currently one of the best paradigms to explain the temperature anisotropy in the CMBR and large scale structures in the Universe. It can occur in many different sectors, such as in the visible sector [31-33], individually or simultaneously [34], and for a review one can read [35]. However, inflation in the gravitational sector is perhaps one of the most natural ways to describe the Universe, first envisaged by Starobinsky [21]. The original model was described by a quadratic curvature action of gravity, and now that we have this infinite derivative modification, which improves the UV aspects of gravity, we should revisit its cosmological properties. $^{4}$

Recently, in [38,39] the authors constructed the most general quadratic curvature, infinite derivative theory of gravity which is free from ghosts and instability around de Sitter and anti-de Sitter backgrounds. ${ }^{5}$ In order to understand the stability of the action around de Sitter and anti-de Sitter backgrounds, the authors of [39] expanded the action up to second order in scalar, vector and tensor modes. It was shown that the only propagating modes would be the scalar and tensor modes.

With the help of these mathematical tools, in [40] and in [41], the authors investigated the scalar and tensor perturbations in an inflationary background for infinite derivative theory of gravity. It was found that in the low energy limit, the scalar and tensor perturbations evolve in exactly the same way as in the Starobinsky model of inflation, but in the UV regime there are some subtle differences, which were highlighted in [41], and we will briefly review them here. We will use the latest bounds on tensor modes to constrain the value of nonlocality $M$. We will also explicitly compute the spectral tilt for the scalar

\footnotetext{
${ }^{4}$ In fact, [36] highlights the robustness of Starobinsky inflation from the quantum corrections point of view for physics beyond the Standard model. [37] showed that under a suitable truncation, infinite derivative gravity can give rise to Starobinsky inflation.

${ }^{5}$ For parity invariant and torsion free gravity.
}

perturbations for the IDG model of inflation for the first time.

\section{INFINITE DERIVATIVE GRAVITY}

The most generic quadratic curvature gravity in 4 dimensions, which can be made ghost free can be written in terms of the Ricci scalar, $R$, the symmetric traceless tensor, $S_{\mu \nu}=R_{\mu \nu}-\frac{1}{4} R g_{\mu \nu}$, analog with the Einstein tensor, $R_{\mu \nu}$ (the Ricci tensor), and the Weyl tensor: $C_{\mu \nu \alpha \beta}$. The $S$ tensor vanishes on maximally symmetric backgrounds $[38]^{6}$ :

$$
\begin{aligned}
S= & \int d^{4} x \sqrt{-g}\left[\frac{M_{P}^{2}}{2} R+\frac{\lambda}{2}\left(R \mathcal{F}_{1}(\square) R\right.\right. \\
& \left.\left.+S_{\mu \nu} \mathcal{F}_{2}(\square) S^{\mu \nu}+C_{\mu \nu \lambda \sigma} \mathcal{F}_{3}(\square) C^{\mu \nu \lambda \sigma}\right)\right],
\end{aligned}
$$

where greek indices $\mu, \nu$ etc. run from 0 to $3, M_{P}^{2}$ is the Planck mass, and $\lambda$ is a dimensional coupling accounting for the higher curvature modification, and the $\mathcal{F}_{i}$ are Taylor expandable (i.e. analytic) functions of the covariant d'Alembertian [5], i.e.

$$
\mathcal{F}_{i}(\square)=\sum_{n=0}^{\infty} c_{i_{n}} \square^{n} / M^{2 n},
$$

where $M$ is the scale of nonlocality and $c_{i_{n}}$ are the coefficients of the series. Using the fact that the $1 / r$ fall of the Newtonian potential continues until around $5 \times 10^{-6} \mathrm{~m}$ [25], we can say that $M>10^{-2} \mathrm{eV}$ [26], a reasonably weak constraint. Previous work on nonlocal theory, without the Weyl term in Eq. (3), used inflation data to estimate $M$ in that simplified version of the theory as $M \sim 10^{15} \mathrm{GeV}$ [19] and $M>10^{8} \mathrm{GeV}$ [42].

In fact, it was already shown in [5], that one can switch off either $\mathcal{F}_{i}(\square)$ in Eq. (1) without loss of generality, i.e. without introducing ghosts in the spectrum and without modifying the graviton propagator in the IR. The full equations of motion for the action have been derived in [43].

For the purpose of investigating inflation, one can use the "redundant functions" method to set $F_{2}(\square)=0$ and therefore study the following action without loss of generality [24]:

$$
\begin{aligned}
S= & \frac{1}{2} \int d^{4} x \sqrt{-g}\left[M_{p}^{2} R+\lambda\left(R \mathcal{F}_{1}(\square) R\right.\right. \\
& \left.\left.+C_{\mu \nu \rho \sigma} \mathcal{F}_{3}(\square) C^{\mu \nu \rho \sigma}\right)\right] .
\end{aligned}
$$

In order to solve the infinite covariant derivatives, one can consider a simple ansatz

\footnotetext{
${ }^{6}$ The original action was written in terms of $R_{\mu \nu}$ and $R_{\mu \nu \lambda \sigma}$ in [5]. However there is no loss of generality in expressing the action as Eq. (1) [38].
} 


$$
\square R=r_{1} R
$$

where $r_{1}$ is a constant, which produces the relation $\mathcal{F}_{1}(\square) R=F_{1} R$, where $F_{1}$ is a constant. ${ }^{7}$

We can now perturb the action around a generic background $\bar{g}_{\mu \nu}$, i.e. $g_{\mu \nu}=\bar{g}_{\mu \nu}+h_{\mu \nu}$, where we can decompose $h_{\mu \nu}$ as follows:

$h_{\mu \nu}=h_{\mu \nu}^{\perp}+\bar{\nabla}_{\mu} A_{\nu}+\bar{\nabla}_{\nu} A_{\mu}+\left(\bar{\nabla}_{\mu} \bar{\nabla}_{\nu}-\frac{1}{4} \bar{g}_{\mu \nu}-\bar{\square}\right) B+\frac{1}{4} \bar{g}_{\mu \nu} h$,

where $h_{\mu \nu}^{\perp}$ is the transverse and traceless spin-2 excitation, $A_{\mu}$ is a transverse vector field, and $(B, h)$ are two scalar degrees of freedom which mix [45]. One can show that the vector mode and the double derivative scalar mode vanish on constant curvature backgrounds [38]. ${ }^{8}$ We are left with two relevant modes, the tensor mode $h_{\mu \nu}^{\perp}$ and $\phi \equiv h-\square B$, i.e.

$$
h_{\mu \nu}=h_{\mu \nu}^{\perp}+\frac{1}{4} g_{\mu \nu} \phi
$$

\section{SCALAR FLUCTUATIONS AROUND INFLATIONARY BACKGROUND}

By inserting Eq. (6) into the action Eq. (3), we find that the scalar part of the quadratic variation of the action is [38]

$$
\delta^{2} S_{0}=\int d^{4} x \sqrt{-g} \frac{\phi}{64}(3 \square+\bar{R})\left[6 \lambda F_{1} \square-M_{p}^{2}\right] \phi,
$$

where $\bar{R}$ is the background Ricci scalar. As we can see, the Weyl tensor term $\mathcal{F}_{3}(\square)$ has no effect here and so the action Eq. (3) should produce the same scalar perturbations as local Starobinsky $R+R^{2}$ inflation, as was shown in [41].

It was shown in $[40,41]$ that then the scalar power spectrum is given by

$$
\left|\delta_{\Phi}(\mathbf{k}, \tau)\right|^{2}=\left.\frac{k^{2}}{16 \pi^{2} a^{2}} \frac{1}{3 \lambda F_{1} \bar{R}}\right|_{k=a H} .
$$

We can calculate the measured power spectrum of the gauge-invariant comoving curvature perturbation $\mathcal{R}$, where in the regime $\dot{H} \ll H^{2}, \mathcal{R} \approx-\frac{H^{2}}{\dot{H}} \Phi$, where dot is defined as derivative with respect to physical time, $t$. Because the Weyl term does not contribute, then at the crossing of the Hubble radius, the scalar power spectrum is the same as in $[40,41]$

\footnotetext{
${ }^{7}$ The more general ansatz $\square R=r_{1} R+r_{2}$, where $r_{1}, r_{2}$ are constants, was used originally in [4] and then [17,18,40,44]. Setting $r_{2}=0$ as we have done here is equivalent to requiring that the cosmological constant $\Lambda$ in the action vanishes.

${ }^{8}$ During inflation the Hubble parameter is nearly constant, so taking the background curvature to be constant is a very good approximation.
}

$$
P_{s}=\left|\delta_{\mathcal{R}}\right|^{2} \approx \frac{H_{k=H a}^{6}}{16 \pi^{2} \dot{H}_{k=H a}^{2}} \frac{1}{3 \lambda F_{1} \bar{R}},
$$

evaluated at $k=a H$. In the above we have multiplied by $H^{4} / \dot{H}^{2}$ in order to transform from the variation of $\Phi$ to the variation of $\mathcal{R}$. We can now recast this in terms of number of $e$-foldings, i.e. $N$, defined at the start of inflation to the end of inflation [35],

$$
N=-\frac{1}{2} \frac{H^{2}}{\dot{H}}
$$

With the help of the above equation, and noting that the background Ricci scalar during inflation is $\bar{R} \approx 12 H^{2}$, which gives us [41]

$$
P_{s}=\left|\delta_{\mathcal{R}}\right|^{2} \approx \frac{N^{2}}{24 \pi^{2}} \frac{1}{6 \lambda F_{1}}
$$

which reduces to the value for Starobinsky inflation [46] when we take the appropriate value for $\lambda F_{1}$, i.e $\lambda F_{1}=1 /\left(6 M_{s}^{2}\right)$, in which case our action reduces to that of Starobinsky.

This is what we expected, because there is no contribution here from the Weyl tensor term. Then using Eq. (11), the scalar spectral tilt is given by ${ }^{9}$

$$
n_{s}=\frac{1}{P_{s}} \frac{d P_{s}}{d N}=1-\frac{2}{N}
$$

where $N$ was defined in Eq. (10). This is the same result as for Starobinsky inflation [27].

\section{TENSOR PERTURBATIONS AROUND INFLATIONARY BACKGROUND}

Tensor modes do not couple to the inflaton field in standard inflation. The sub-Hubble tensor modes describe free gravitational waves inside the Hubble patch, which during inflation are carried outside the Hubble patch so that on super-Hubble scales they are locked in. We will now calculate the tensor perturbations for our action. While the addition of the Weyl term does not affect the scalar perturbations, it does affect the tensor perturbations. When we insert Eq. (6) into the action Eq. (3), then the tensor part of the quadratic variation of the action is [38 $]^{10}$

\footnotetext{
${ }^{9}$ Normally $n_{s}$ is given in terms of $k$ as $n_{s}=\frac{d\left(\ln P_{s}\right)}{d(\ln k)}$, but we write it in terms of $N=\ln (a H)$ using $\frac{d\left(\ln P_{s}\right)}{d(\ln k)}=\frac{1}{P_{s}} \frac{d P_{s}}{d N} \frac{d N}{d(\ln k)}$ and then noting that $\frac{d N}{d(\ln k)}=\frac{d(\ln a H)}{d(\ln a H)}=1$.

${ }^{10}$ Note that compared to [38], we took $\mathcal{F}_{2}(\square)=0$ and $f_{1_{0}}=F_{1}$.
} 


$$
\begin{aligned}
\delta^{2} S_{\perp}= & \frac{\lambda}{4} \int d^{4} x \sqrt{-\bar{g}} h_{\mu \nu}^{\perp}\left(\square-\frac{\bar{R}}{6}\right) F_{1} \bar{R} \\
& \times\left[1+\frac{1}{F_{1} \bar{R}}\left(\square-\frac{\bar{R}}{3}\right) \mathcal{F}_{3}\left(\square+\frac{\bar{R}}{3}\right)\right] h^{\perp \mu \nu} .
\end{aligned}
$$

The result Eq. (13) has the standard pole of the propagator at $\square=\frac{\bar{R}}{6}$. Eq. (13) is simply the result for an action of type $R+\lambda F_{1} R^{2}$, multiplied by the extra factor in the square brackets. In order to not introduce any ghosts into the propagator, we require that there are no extra poles resulting from this term in square brackets. The obvious choice is the exponential of an entire function, which by definition has no roots. We therefore define [41]

$$
P(\square) \equiv 1+\frac{1}{F_{1} \bar{R}}\left(\square-\frac{\bar{R}}{3}\right) \mathcal{F}_{3}\left(\square+\frac{\bar{R}}{3}\right),
$$

where $P(\square)$ is the exponential of an entire function. We now look at the precise form of $P(\square)$. If we take the simplest choice, $P(\square)=e^{\omega(\square)}$, then we find that

$$
\mathcal{F}_{3}(\square)=F_{1} \bar{R} \frac{e^{\omega(\square-\bar{R} / 3)}-1}{\square-\frac{2}{3} \bar{R}} .
$$

However, this gives us a pole in $F_{3}(\square)$ at $\square=2 \bar{R} / 3$. $^{11}$ The simplest choice which avoids this pole is [41]

$$
\mathcal{F}_{3}(\square)=F_{1} \bar{R} \frac{e^{H\left(\square-\frac{2}{3} \bar{R}\right)}-1}{\square-\frac{2}{3} \bar{R}},
$$

where $H(\square)$ is an entire function. Then combining Eq. (14) with this choice means that

$$
P(\square)=e^{H(\square-\bar{R} / 3)} .
$$

The extra exponential factor is always positive and will be very important when we look at the scalar-tensor ratio and will allow us to put a constraint on the scale of nonlocality. The tensor power spectrum for the action Eq. (3) is therefore multiplied by $P(\square)$ evaluated at $\square=\bar{R} / 6$, the root of Eq. (13). Therefore the power spectrum becomes [41]

$$
\left|\delta_{h}\right|^{2}=\frac{H^{2}}{2 \pi^{2} \lambda F_{1} \bar{R}} e^{H(-\bar{R} / 6)}
$$

and the ratio between the tensor and scalar power spectrums can be given by

\footnotetext{
${ }^{11} \mathrm{~A}$ pole in $F_{3}(\square)$ is not necessarily disastrous for the theory, because the propagators are still well defined, but having a function $F_{3}(\square)$ which is analytic shows that the theory is well constructed.
}

$$
\begin{aligned}
r & =\frac{2\left|\delta_{h}\right|^{2}}{\left|\delta_{R}\right|^{2}} \\
& =48 H^{2} e^{H(-\bar{R} / 6)} \frac{\dot{H}^{2}}{H^{4}},
\end{aligned}
$$

(where the factor of 2 accounts for the two polarizations of the tensor modes). We can write this tensor-scalar ratio using the definition of the number of $e$-foldings $N$ Eq. (10) as

$$
r=\frac{12}{N^{2}} e^{H(-\bar{R} / 6)} .
$$

When we compare this to the ratio given by Starobinsky inflation, where

$$
r=\frac{12}{N^{2}},
$$

we see that there is an extra modulating exponential factor $e^{H(-\bar{R} / 6)}$, which was defined in Eq. (14), due to the addition of nonlocal gravity.

\section{SCALAR TO TENSOR RATIO, $r$, AND CONSTRAINING THE SCALE OF NONLOCALITY}

From the 2015 Planck data given in [23], the bound on tensor-to-scalar ratio is given by $r<0.07$, and so from Eq. (20), we find that

$$
\frac{12}{N^{2}} e^{H(-\bar{R} / 6)}<0.07 .
$$

When we take the logarithm of Eq. (22), we obtain the constraint

$$
H(-\bar{R} / 6)<2 \log (N)-5.14,
$$

and during inflation, we obtain

$$
\bar{R} \approx 12 H^{2} \sim \frac{9.02 \times 10^{32}}{N^{2}} \mathrm{GeV}^{2} .
$$

Therefore Eq. (23) becomes

$$
H\left(-\frac{9.02 \times 10^{32} \mathrm{GeV}^{2}}{6 M^{2} N^{2}}\right)<2 \log (N)-5.14 .
$$

Of course, as far as the form of $H(\square-\bar{R} / 3)$ in Eq. (17) is concerned, it could be any entire function, as long as it retains that in momentum space, $H\left(-k^{2}-\bar{R} / 3\right) \rightarrow \infty$, when we take the UV limit $k^{2} \rightarrow \infty$. This allows the UV propagator to be exponentially suppressed. This requirement implies that $H$ must have terms of the form $(-1)^{n}(\square-\bar{R} / 3)^{n}$ due to the fact that $\square$ becomes $-k^{2}$ in momentum space. One can take some simple polynomial functions to see what the effect will be on the scalar-tensor ratio. Note that when we evaluate $r$ at $\square=\bar{R} / 6$, 
these terms become $(-1)^{n}(\bar{R} / 6-\bar{R} / 3)^{n}=(\bar{R} / 6)^{n}$. As this is strictly positive, this will gives us an increased value of $r$ compared to that given for Starobinsky inflation in Eq. (21), which means we can then constrain $M$ using the upper bound on $r$ from the Planck data. We will now take

(i) $\left.H\left(-\frac{\bar{R}}{6}\right)=(-1)^{n}(\square-\bar{R} / 3) M^{2}\right)\left.^{n}\right|_{\square=\bar{R} / 6}=\left(\bar{R} / 6 M^{2}\right)^{n}$ We wish to seek a bound on $M$, which translates Eq. (25) into ${ }^{12}$

$$
M>\frac{\sqrt{\frac{3}{2}}}{N}[(2 \log (N)-5.14)]^{-1 / 2 n} \times 10^{16} \mathrm{GeV} .
$$

If we take $H(-\bar{R} / 6)=-(\square-\bar{R} / 3) /\left.M^{2}\right|_{\square=\bar{R} / 6}$, as well as $r<0.07$ and $N=60 e$-foldings, then

$$
M>1.17 \times 10^{14} \mathrm{GeV} .
$$

If we take $H(-\bar{R} / 6)=(\square-\bar{R} / 3)^{2} /\left.M^{4}\right|_{\square=\bar{R} / 6}$, $r<0.07$ and $N=60 e$-foldings, then

$$
M>1.55 \times 10^{14} \mathrm{GeV} .
$$

If we take $H(-\bar{R} / 6)=(\square-\bar{R} / 3)^{2 n} /\left.M^{2 n}\right|_{\square=\bar{R} / 6}$, we obtain

$$
M>2.04 \times(0.573)^{1 / n} \times 10^{14} \mathrm{GeV} .
$$

(ii) $H\left(-\frac{\bar{R}}{6}\right)=-\left(\square-\frac{\bar{R}}{3}\right) / M^{2}+(-1)^{n}\left(\square-\frac{\bar{R}}{3}\right)^{a} /\left.M^{2 a}\right|_{\square=\bar{R} / 6}$, Let us illustrate the more general situation, when $H(\square)$ is a binomial. There is an extra degree of freedom here because the coefficients in front of the two terms could be different, but to keep things simple we have assumed that they are the same. In which case, using that $r<0.07$ and $N=60$, our constraint Eq. (23) becomes

$$
\frac{9.02 \times 10^{32} \mathrm{Gev}}{6 M^{2} 60^{2}}+\left(\frac{9.02 \times 10^{32} \mathrm{Gev}}{6 M^{2} 60^{2}}\right)^{a}<3.049 \text {. }
$$

This gives us a lower bound on $M$ of $1.78 \times$ $10^{14} \mathrm{GeV}$ for $a=2$, rising to $1.96 \times 10^{14} \mathrm{GeV}$ for $a=16$ and $2.03 \times 10^{14} \mathrm{GeV}$ for $a=64$.

(iii) $H\left(-\frac{\bar{R}}{6}\right)=\sum_{a=1}^{\infty}(-1)^{a}(\square-\bar{R} / 3)^{a} /\left.M^{2 a}\right|_{\square=\bar{R} / 6}$ : Finally we take the case where $H$ is a sum of $\square$ over all orders, again assuming that the coefficients of the terms are the same. Then our Eq. (23) becomes

\footnotetext{
${ }^{12}$ Note that $50<N<60$ and $2 \log (N) \geq 2 \log (50)=7.9$ so therefore $2 \log (N)-5.14 \geq 0$.
}

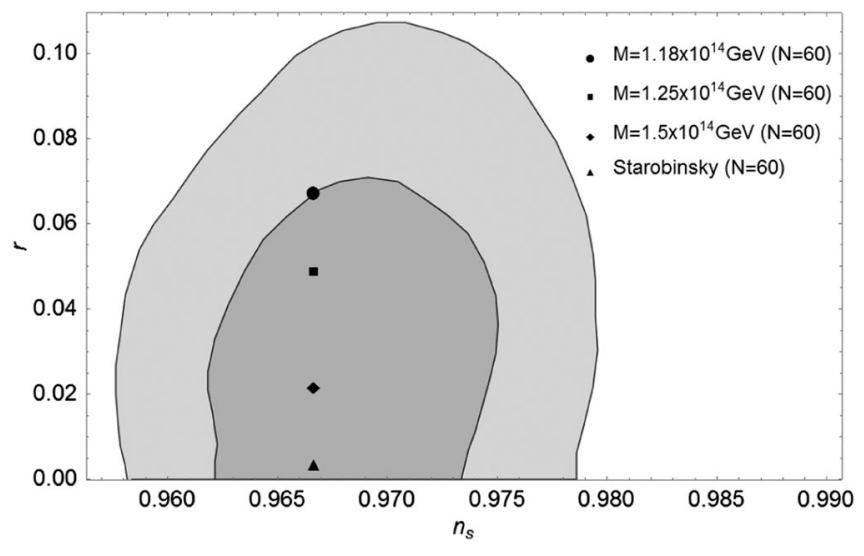

FIG. 1. A plot of the tensor-scalar ratio $r$ vs the spectral index $n_{s}$ for different values of $M$ where we have taken $H(-\bar{R} / 6)=$ $-(\square-\bar{R} / 3) /\left.M^{2}\right|_{\square=\bar{R} / 6}$ using Eq. (32). We have taken $N=60$ and also plotted the 2015 Planck data.

$$
\sum_{a=1}^{\infty}\left(\frac{9.02 \times 10^{32} \mathrm{Gev}}{6 \times 60^{2} M^{2}}\right)^{2 a}<30.49
$$

Numerically, this gives us a constraint of $M>2.35 \times 10^{14} \mathrm{GeV}$.

(iv) So far we have taken only forms of $P(\square)$ which give a value larger than 1 for $P(\square=\bar{R} / 6)$, but it is also possible for us to choose a form of $P(\square)$ such that $P(\bar{R} / 6)$ is less than $1 .{ }^{13}$ Using Eq. (20), a negative value of for the argument of the exponential gives a lower tensor-scalar ratio than Starobinsky inflation in Eq. (21), and because we do not currently have a lower bound on $r$, this does not provide us with any constraint on $M$. If future data shows that $r$ is lower than that predicted by Starobinsky, then this could prove a useful model. ${ }^{14}$

\section{VI. $r$ VS $n_{s}$ PLOT FOR UV COMPLETE STAROBINSKY MODEL OF INFLATION}

If we want to plot $r$ against $n_{s}$, then we should note using Eq. (12), Eq. (20) that for nonlocal gravity

$$
\begin{aligned}
r & =\frac{12}{N^{2}} e^{H(\bar{R} / 6)} \\
& =3\left(1-n_{s}\right)^{2} e^{H(-\bar{R} / 6)} .
\end{aligned}
$$

We can plot this for different forms of $H(\square)$ and compare with the 2015 Planck data at the $68 \%$ and $95 \%$ confidence

\footnotetext{
${ }^{13}$ For example by taking $P(\square)=\exp [\square(\square-\bar{R} / 3)]$. This still ensures that we have no pole in $F_{3}(\square)$, but gives us a negative value for the argument of the exponential when we evaluate $P(\square)$ at $\square=\bar{R} / 6$.

${ }^{14} \mathrm{We}$ could also take $P(\square)=e^{(\square-\bar{R} / 6)(\square-\bar{R} / 3)}$. When we evaluate this at $\square=\bar{R} / 6$, then this gives us the same prediction as Starobinsky.
} 
TABLE I. A table of the tensor-scalar ratio for various values of the scale of nonlocality $M$, using Eq. (24) and Eq. (32) and taking $H(-\bar{R} / 6)=-(\square-\bar{R} / 3) /\left.M^{2}\right|_{\square=\bar{R} / 6}$. In the fifth line, we have taken the limit $M \rightarrow \infty$, which means that our action reduces to that of Starobinsky. The tensor-scalar ratio for $M=M_{p}$ is a factor of $\exp \left(7.04 \times 10^{-9}\right)$ bigger than for Starobinsky inflation, i.e. when $M \rightarrow \infty$. This factor is equal to 1 to nine significant figures, i.e. for $M=M_{p}$, the action Eq. (3) is effectively indistinguishable from Starobinsky inflation.

\begin{tabular}{lc}
\hline$M$ & Tensor-Scalar Ratio \\
\hline $1 \times 10^{14} \mathrm{GeV}$ & 0.22 \\
$1.5 \times 10^{14} \mathrm{GeV}$ & 0.021 \\
$2.0 \times 10^{14} \mathrm{GeV}$ & 0.0094 \\
$M_{p}=2.435 \times 10^{18} \mathrm{GeV}$ & 0.0033 \\
$\infty$ (Starobinsky) & 0.0033 \\
\hline \hline
\end{tabular}

level from [23] for $N=60$. If we take $H(\square)=-\square / M^{2}$, then in Fig. [1] this gives us a constraint on $M$ of

$$
M>1.18 \times 10^{14} \mathrm{GeV} .
$$

Finally we calculate the tensor-scalar ratio for various values of the scale of nonlocality $M$ in [Table I].

\section{CONCLUSION}

In recent years, an infinite derivative, ghost-free, quadratic curvature action of gravity has been shown to solve the singularity problem and give a universal prediction for the Newtonian potential at large distances.
Using the scalar spectral index and the tensor-scalar ratio together with the latest Planck data, we have found a constraint on the value of the scale of nonlocality for various cases of IDG.

Using the simplest case of IDG which avoids poles throughout the theory, this provides us with a much stronger constraint than before, of $M>1.18 \times 10^{14} \mathrm{GeV}$ (around $10^{-4} M_{P}$ ) using cosmological data whereas previously our best constraint from below using the full theory was $10^{-2} \mathrm{eV}$ using data from laboratory experiments. Using a reduced version of the action, the constraints $M>10^{8} \mathrm{GeV}$ [19] and $M \sim 10^{15} \mathrm{GeV}$ [42] were found using cosmological data by other authors, which is consistent with our result.

We have also looked at different classes of the theory, with different versions of the entire function, and obtained modified constraints within these versions. In principle we can take a different argument of the function, which will give us different values of the tensor-scalar ratio.

Our result allows us to compare experimentally IDG with Starobinsky inflation, and can provide an explanation for possible differences between the predictions of Starobinsky inflation and cosmological data. With further data on the scalar-tensor ratio we will be able to constrain the scale of nonlocality even further.

\section{ACKNOWLEDGMENTS}

We would like to thank Alexey Koshelev and Anupam Mazumdar for valuable discussions and guidance, without which this paper would not have been possible.
[1] C. M. Will, The confrontation between general relativity and experiment, Living Rev. Relativ. 17, 4 (2014).

[2] K. S. Stelle, Renormalization of higher derivative quantum gravity, Phys. Rev. D 16, 953 (1977).

[3] P. Van Nieuwenhuizen, On ghost-free tensor Lagrangians and linearized gravitation, Nucl. Phys. B60, 478 (1973).

[4] T. Biswas, A. Mazumdar, and W. Siegel, Bouncing universes in string-inspired gravity, J. Cosmol. Astropart. Phys. 03 (2006) 009.

[5] T. Biswas, E. Gerwick, T. Koivisto, and A. Mazumdar, Towards Singularity- and Ghost-Free Theories of Gravity, Phys. Rev. Lett. 108, 031101 (2012).

[6] E. Tomboulis, Renormalizability and asymptotic freedom in quantum gravity, Phys. Lett. 97B, 77 (1980); Superrenormalizable gauge and gravitational theories, arXiv:hep-th/ 9702146; Nonlocal and quasilocal field theories, Phys. Rev. D 92, 125037 (2015).

[7] L. Modesto, Super-renormalizable quantum gravity, Phys. Rev. D 86, 044005 (2012).
[8] S. Talaganis, T. Biswas, and A. Mazumdar, Towards understanding the ultraviolet behavior of quantum loops in infinite-derivative theories of gravity, Classical Quantum Gravity 32, 215017 (2015).

[9] S. Talaganis and A. Mazumdar, High-energy scatterings in infinite-derivative field theory and ghost-free gravity, Classical Quantum Gravity 33, 145005 (2016).

[10] T. Biswas, T. Koivisto, and A. Mazumdar, Nonlocal theories of gravity: The flat space propagator, arXiv: 1302.0532.

[11] T. Biswas, J. A. R. Cembranos, and J. I. Kapusta, Thermal Duality and Hagedorn Transition from $p$-adic Strings, Phys. Rev. Lett. 104, 021601 (2010); Thermodynamics and cosmological constant of non-local field theories from p-adic strings, J. High Energy Phys. 10 (2010) 048; Finite temperature solitons in nonlocal field theories from $p$-adic strings, Phys. Rev. D 82, 085028 (2010).

[12] T. Biswas and N. Okada, Towards LHC physics with nonlocal standard model, Nucl. Phys. B898, 113 (2015). 
[13] A. A. Tseytlin, On singularities of spherically symmetric backgrounds in string theory, Phys. Lett. B 363, 223 (1995).

[14] W. Siegel, Stringy gravity at short distances, arXiv:hep-th/ 0309093.

[15] V. P. Frolov and A. Zelnikov, Head-on collision of ultrarelativistic particles in ghost-free theories of gravity, arXiv: 1509.03336; V. P. Frolov, Mass Gap for Black-Hole Formation in Higher-Derivative and Ghost-Free Gravity, Phys. Rev. Lett. 115, 051102 (2015); V. P. Frolov, A. Zelnikov, and T. de Paula Netto, Spherical collapse of small masses in the ghost-free gravity, J. High Energy Phys. 06 (2015) 107.

[16] A. Conroy, A. S. Koshelev, and A. Mazumdar, Geodesic completeness and homogeneity condition for cosmic inflation, Phys. Rev. D 90, 123525 (2014).

[17] T. Biswas, T. Koivisto, and A. Mazumdar, Towards a resolution of the cosmological singularity in non-local higher derivative theories of gravity, J. Cosmol. Astropart. Phys. 11 (2010) 008.

[18] T. Biswas, A. S. Koshelev, A. Mazumdar, and S. Y. Vernov, Stable bounce and inflation in non-local higher derivative cosmology, J. Cosmol. Astropart. Phys. 08 (2012) 024.

[19] T. Biswas and A. Mazumdar, Super-inflation, non-singular bounce, and low multipoles, Classical Quantum Gravity 31, 025019 (2014).

[20] D. Chialva and A. Mazumdar, Cosmological implications of quantum corrections and higher-derivative extension, Mod. Phys. Lett. A 30A, 1540008 (2015).

[21] A. A. Starobinsky, A new type of isotropic cosmological models without singularity, Phys. Lett. 91B, 99 (1980).

[22] C. L. Bennett, D. Larson, J. L. Weiland, N. Jarosik, G. Hinshaw, N. Odegard, K. M. Smith, R. S. Hill et al., Nine-year wilkinson microwave anisotropy probe (wmap) observations: final maps and results, Astrophys. J. Suppl. Ser. 208, 20 (2013).

[23] P. A. R. Ade et al. (Planck Collaboration), Planck 2015 results. XIII. Cosmological parameters, Astron. Astrophys. 594, A13 (2016).

[24] A. Conroy, A. S. Koshelev, and A. Mazumdar, Defocusing of null rays in infinite derivative gravity, J. Cosmol. Astropart. Phys. 01 (2017) 017.

[25] D. J. Kapner, T. S. Cook, E. G. Adelberger, J. H. Gundlach, B. R. Heckel, C. D. Hoyle, and H. E. Swanson, Tests of the Gravitational Inverse-Square Law Below the Dark-Energy Length Scale, Phys. Rev. Lett. 98, 021101 (2007).

[26] J. Edholm, A. S. Koshelev, and A. Mazumdar, Behavior of the Newtonian potential for ghost-free gravity and singularity-free gravity, Phys. Rev. D 94, 104033 (2016).

[27] V. F. Mukhanov, H. A. Feldman, and R. H. Brandenberger, Theory of cosmological perturbations. Part 1. Classical perturbations. Part 2. Quantum theory of perturbations. Part 3. Extensions, Phys. Rep. 215, 203 (1992).

[28] A. H. Guth, The inflationary universe: A possible solution to the horizon and flatness problems, Phys. Rev. D 23, 347 (1981).
[29] A. D. Linde, A new inflationary universe scenario: A possible solution of the horizon, flatness, homogeneity, isotropy and primordial monopole problems, Phys. Lett. 108B (1982) 389.

[30] A. Albrecht and P. J. Steinhardt, Cosmology for Grand Unified Theories with Radiatively Induced Symmetry Breaking, Phys. Rev. Lett. 48, 1220 (1982).

[31] D. La and P. J. Steinhardt, Extended Inflationary Cosmology, Phys. Rev. Lett. 62, 376 (1989); Erratum, Phys. Rev. Lett. 62, 1066(E) (1989).

[32] F. L. Bezrukov and M. Shaposhnikov, The standard model Higgs boson as the inflaton, Phys. Lett. B 659 , 703 (2008).

[33] R. Allahverdi, K. Enqvist, J. Garcia-Bellido, and A. Mazumdar, Gauge Invariant MSSM Inflaton, Phys. Rev. Lett. 97, 191304 (2006).

[34] A. R. Liddle, A. Mazumdar, and F. E. Schunck, Assisted inflation, Phys. Rev. D 58, 061301 (1998).

[35] A. Mazumdar and J. Rocher, Particle physics models of inflation and curvaton scenarios, Phys. Rep. 497, 85 (2011).

[36] A. Salvio and A. Mazumdar, Classical and quantum initial conditions for Higgs inflation, Phys. Lett. B 750, 194 (2015); Higgs stability and the $750 \mathrm{GeV}$ diphoton excess, Phys. Lett. B 755, 469 (2016).

[37] F. Briscese, A. Marcian, L. Modesto, and E. N. Saridakis, Inflation in (super-)renormalizable gravity, Phys. Rev. D 87, 083507 (2013).

[38] T. Biswas, A. S. Koshelev, and A. Mazumdar, Gravitational theories with stable (anti-)de Sitter backgrounds, Fundam. Theor. Phys. 183, 97 (2016).

[39] T. Biswas, A. S. Koshelev, and A. Mazumdar, Consistent higher derivative gravitational theories with stable de Sitter and anti-de Sitter backgrounds, arXiv:1606.01250.

[40] B. Craps, T. De Jonckheere, and A. S. Koshelev, Cosmological perturbations in non-local higher-derivative gravity, J. Cosmol. Astropart. Phys. 11 (2014) 022.

[41] A. S. Koshelev, L. Modesto, L. Rachwal, and A. A. Starobinsky, Occurrence of exact $R^{2}$ inflation in nonlocal UV-complete gravity, J. High Energy Phys. 11 (2016) 067.

[42] T. Biswas, R. Brandenberger, A. Mazumdar, and W. Siegel, Non-perturbative gravity, Hagedorn bounce and CMB, J. Cosmol. Astropart. Phys. 12 (2007) 011.

[43] T. Biswas, A. Conroy, A. S. Koshelev, and A. Mazumdar, Generalized ghost-free quadratic curvature gravity, Classical Quantum Gravity 31, 015022 (2014); Erratum: Generalized ghost-free quadratic curvature gravity, Classical Quantum Gravity 31, 159501(E) (2014).

[44] S. Deser and R. P. Woodard, Observational viability and stability of nonlocal cosmology, J. Cosmol. Astropart. Phys. 11 (2013) 036.

[45] E. D'Hoker, D. Z. Freedman, S. D. Mathur, A. Matusis, and L. Rastelli, Graviton exchange and complete four point functions in the AdS/CFT correspondence, Nucl. Phys. B562, 353 (1999).

[46] Q. G. Huang, A polynomial f(R) inflation model, J. Cosmol. Astropart. Phys. 02 (2014) 035. 